\title{
European land-use at 6000 BP: from on-site data to the large-scale view
}

\author{
Nicki Whitehouse', M. Madella² and F. Antolín ${ }^{3}$
}

Barcelona, Spain, 21-23 May 2018

The LandCover6k working group investigates whether prehistoric and historic human impacts on land cover (i.e. anthropogenic land-cover change due to land use - LULC) were sufficiently large to have a significant impact on regional climates. This workshop focused on reconstructing land use for the European continent by synthesizing farming and landscape management patterns derived from the archaeological record, for a $6000 \mathrm{BP}$ time slice. We were also interested in the location of settlements, field systems, and industrial activities. The main objectives of the workshop were to:

(i) Review LU classifications developed for other regions (Morrison et al. 2018), ensure these are usable for Europe at $6000 \mathrm{BP}$, and develop geography-specific lower-classification categories;

(ii) Establish regions with good datasets for LU and produce preliminary LU maps following the methodology of Morrison et al. (2018);

(iii) Identify high-resolution case-study areas for detailed LU mapping;

(iv) Agree on a publication strategy and subsequent analyses.

\section{Workshop outcomes}

Twenty-two European archaeobotanists, archaeozoologists, chronologists, GIS, climate and land-use modelers attended the workshop. In addition, some researchers joined the meeting remotely via an internet stream, or sent materials, and others have been approached since the meeting to contribute data from under-represented regions.

The first day and a half was devoted to expectations of the archaeological and modeling communities for the land-use development and best approaches towards synthesizing and reconstructing land use at a continental scale. There was considerable enthusiasm from the European community to be involved and contribute data. It was agreed that land maps should be developed from archaeological-site data points and then aggregated at a sub-regional level. Metadata should be fully accessible and traceable, and underpinned by high quality chronological data. This will allow an evaluation of uncertainty, ensure the work is archaeologically accurate and allow future revisions. A European database of ${ }^{14} \mathrm{C}$ dates developed by Marc Vander Linden (University of Cambridge, UK) was made available to facilitate mapping. Discussion

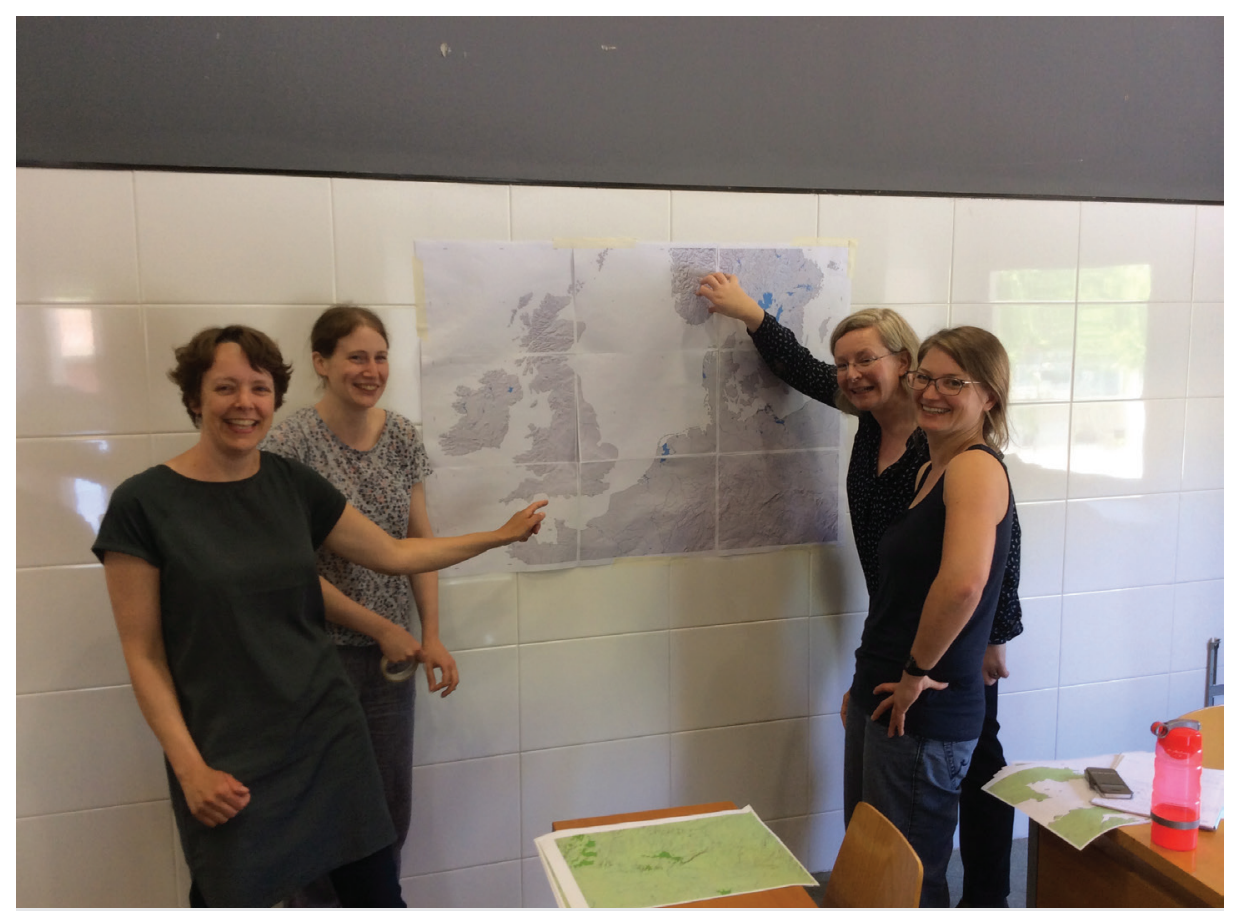

Figure 1: Participants from the Northern Europe Group mapping their region. Photo credit: Welmoeud Out.

also focused around "rules for interpolation" between data points and "unknown" areas, data quality and traceability. Each LU area should be assigned a percentage land-use value as far as possible, allocated at a regional or country level, based on expert knowledge; proportions of crops and animals will be assessed for particular regions.

There were extended discussions on LU categories (Morrison et al. 2018), which led to the addition of a LU category for "no evidence" and adjustments to lower-level categories for hunting-foraging-fishing, pastoralism and agriculture to fully reflect the European evidence. Further work around definitions will be needed. The final half-day of the workshop was spent undertaking initial top-level mapping on printed versions of gridded maps.

The main objectives of the workshop were met; more time was focused on item (i) than initially envisaged. However, this allowed the group to develop the necessary protocols for step (ii) and (iii). We are now progressing with step (ii) and have established areas for which there is good data coverage (iii). Datasets are well distributed across Europe, but further work will be needed to fill in data gaps and develop protocols for data quality.

\section{Next steps}

Maps and ${ }^{14} \mathrm{C}$ datasets have been transferred into a simple GIS database to produce preliminary LU maps. These will need considerable work and refining. A follow-up meeting is planned for 29-30 January 2019 at the Research Center for Wetland Archaeology, in Hemmenhofen, Germany.

We are keen for interested archaeologists to join the group, especially from regions that are currently less-well represented (e.g. France, some areas of North and central Eastern Europe, and southern parts of the Mediterranean). Visit our website for more information: pastglobalchanges.org/ini/wg/ landcoverbk

\section{AFFILIATIONS}

${ }^{1}$ School of Geography, Earth and Environmental Sciences, Plymouth University, UK

${ }^{2}$ ICREA and Department of Humanities, Universitat Pompeu Fabra, Barcelona, Spain

${ }^{3}$ Integrative Prehistory and Archaeological Science (IPAS), University of Basel, Switzerland

\section{CONTACT}

Nicki Whitehouse: Nicola.whitehouse@plymouth.ac.uk REFERENCES

Morrison KD et al. (2018) PAGES Mag 26: 8-9 